\title{
A Third hand for array tomography
}

\author{
Eric Hanssen ${ }^{1}$ \\ 1. Advanced Microscopy Facility, Bio21 Molecular Science and Biotechnology Institute, The University \\ of Melbourne, VIC 3010, Australia
}

With technology advancing at a rapid pace and electron microscope manufacturer listening to the scientific community the innovation have been numerous on several fronts in the electron microscopy world. The main one is the commercialisation of direct detectors for structural and cellular biology. But one of the less mediatised advances is the automation of the microscopes themselves. Whether it is TEM or SEM most major manufacturer now commercialise tools with fully automated alignment and most also have automated acquisition. With these two innovations the operator is rendered almost irrelevant for the collection of data.

With an increase interest in 3D electron microscopy and the automation of microscopes array tomography is currently one of the skyrocketing field of electron microscopy. It is mainly based on the acquisition of serial sections and the acquisition either in a TEM or a SEM of the same feature of interest in each section. In an era where one needs minimum skills to observe one's samples on an electron microscope and where the micrographs are acquired automatically the preparation of the sample is one of the limiting factors. The skill set required to collect serial section takes weeks to months to acquire and is mainly dictated by hand/eye coordination and steadiness of movement. The recovery of few serial sections is achievable by most, several dozen is only achieved by a small minority and only a handful of microscopist around the world can recover several hundreds.

The advent of 3D printing, if not quite yet in every household, is changing the way we at least do science and how we prepare some experiments, especially in engineering. This technology is now available in pretty much every university around the world and accessible by staff and students for free or only few dollars.

This paper describe a new simple 3D printed tool (Figure 1) to help the recovery of serial section on any support for array tomography backscattered imaging, TEM serial tomography or any other technique requiring serial sections. The $3 \mathrm{D}$ print only needs few bolts and nuts that can be found in any hardware store (4 M4x65mm and M4x20mm bolts as well as $5 \mathrm{M} 4$ nuts). We also give the download link for .stl file of the the tool which can be directly 3D printed (Figure 2 left) or transformed in a CAD file for machining (Figure 2 right). 


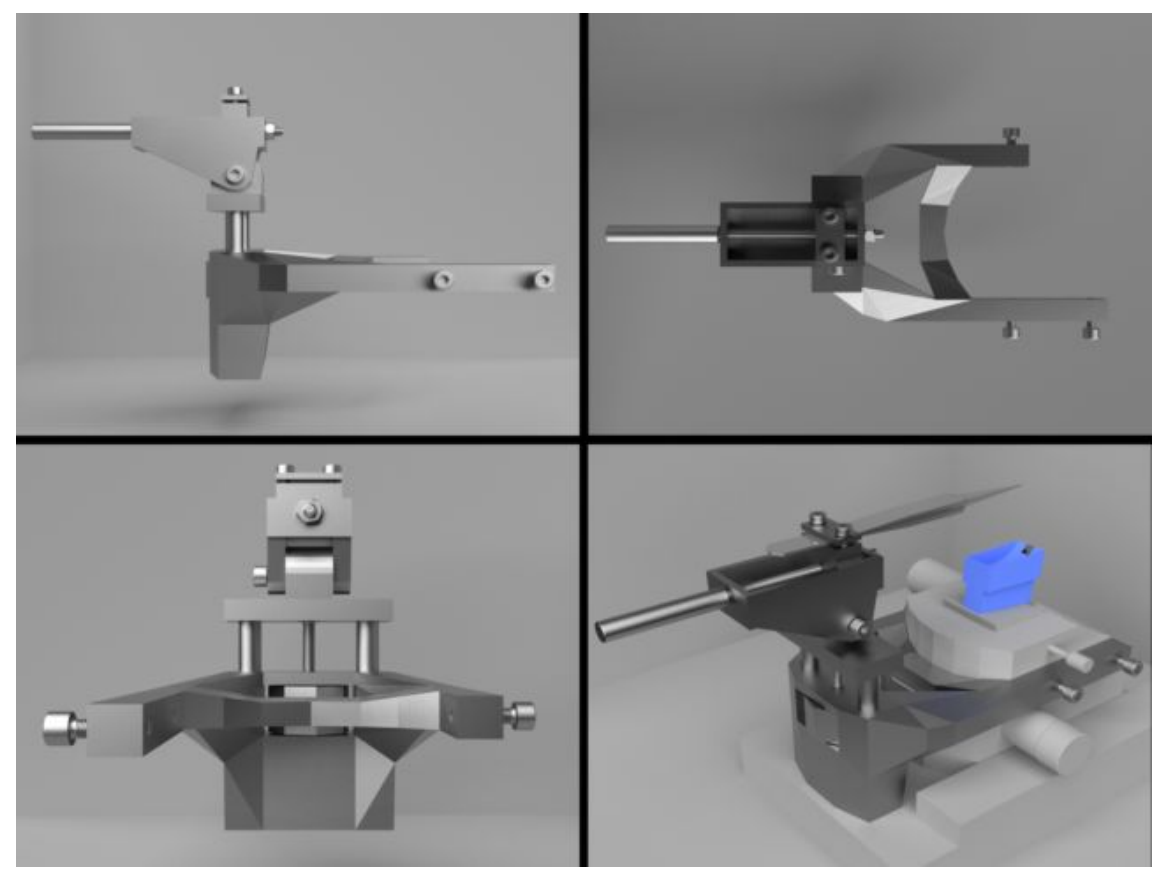

Figure 1: CAD drawing render of the micromanipulator with clamping on ultramicrotome knife block

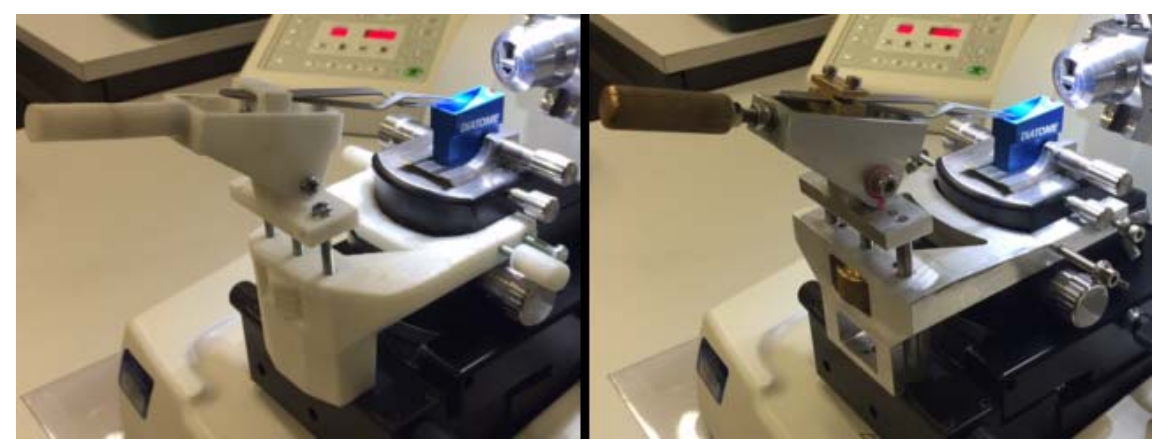

Figure 2: Left, 3D printed version install on a Leica UC7, the design should be compatible with several brand sof ultramicrotome. Right, CNC machined version of the tool on the UC7.

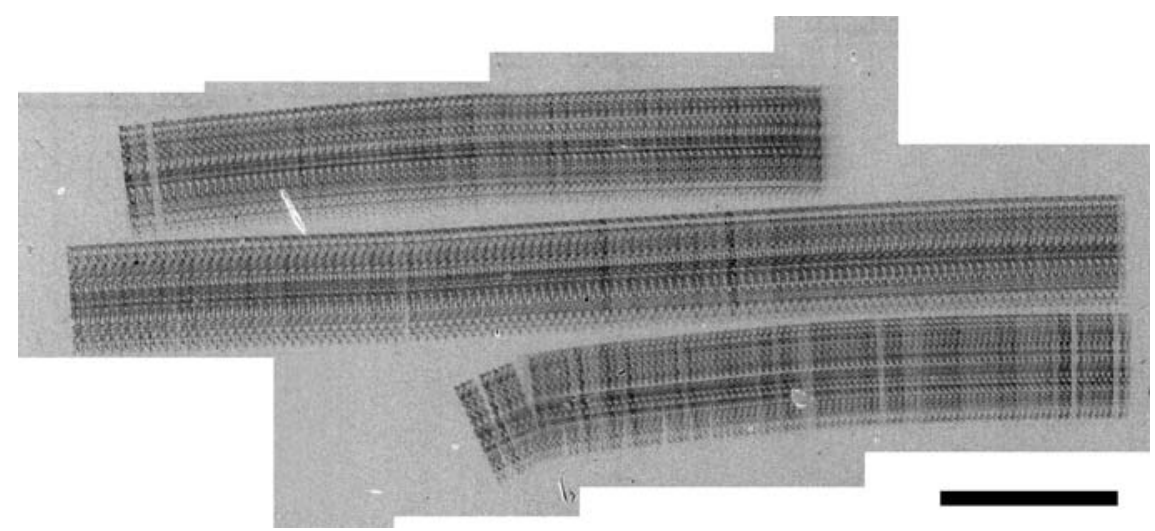

Figure 3: Backscattered SEM image of 350 serial sections collected on a Thermanox (NUNC) coverslip. Scale bar $1 \mathrm{~mm}$. 